\title{
Far-infrared vibrational modes of polycrystalline saccharides
}

\author{
P.C. Upadhya ${ }^{a}$, Y.C. Shen ${ }^{\text {a }}$, A.G. Davies ${ }^{b}$, E.H. Linfield ${ }^{a}{ }^{*}$ \\ ${ }^{a}$ Cavendish Laboratory, University of Cambridge, Madingley Road, Cambridge CB3 OHE, UK \\ ${ }^{\mathrm{b}}$ School of Electronic and Electrical Engineering, University of Leeds, Leeds LS2 9JT, UK
}

Received 16 October 2003; received in revised form 10 December 2003; accepted 10 December 2003

\begin{abstract}
We have measured the temperature dependence of the far-infrared vibrational modes of polycrystalline saccharide molecules in the frequency range $0.1-3.0 \mathrm{THz}$ using a time-resolved terahertz spectroscopy system. Coherent measurement of the transmitted terahertz electric fields showed distinct absorption features, in both monosaccharides and disaccharides, originating from inter- and intra-molecular vibrational modes. These results show that far-infrared absorption features are highly sensitive to the structure and spatial arrangement of molecules. Furthermore, we demonstrate a broad band terahertz spectroscopy system that can be used to probe molecular interactions up to higher frequencies $(>6.0 \mathrm{THz})$.
\end{abstract}

(C) 2004 Elsevier B.V. All rights reserved.

Keywords: Terahertz; Time-domain spectroscopy; Monosaccharide; Disaccharide

\section{Introduction}

The large organic molecules, which are most abundant in all cells, are the carbohydrates, lipids, proteins and nucleic acids. The assembly of these complex macromolecules is from smaller organic building blocks, which are referred to as biomolecular precursors (monomers). They include the simple sugars, the fatty acids, the amino acids, and the nucleotides, and each has been found in all known cells and living systems. The essential feature of these monomer precursors is their capacity to form chemical bonds to at least two other monomer precursors thereby forming linear, chainlike polymers, as well as cross-linked, network polymeric products. The resulting molecular structures are critically important to biological function. Low frequency vibrational spectroscopy could potentially provide information on molecular structure, spatial orientations, and the nature of biological activity [1-4]. Indeed, there have been both experimental and theoretical studies using infrared (IR) and Raman spectroscopy of nucleic acid bases [5], amides [6] and glucose [7,8], and these have revealed details of the molecular dynamics in the far- and mid-infrared spectral ranges. Terahertz time-domain spectroscopy has also proved

\footnotetext{
* Corresponding author. Tel.: +44-1223-764165; fax: +44-1223-337271.

E-mail addresses: pcu20@cam.ac.uk (P.C. Upadhya), eh110@cam.ac.uk (E.H. Linfield).
}

to be a versatile tool for quantitative detection and conformational analysis of bio-molecules, specifically, at low frequencies [2,9]. To date, though, there have only been a few terahertz investigations on large molecules of biological relevance owing to the dearth of technologies to access this spectral range and a lack of theoretical models to interpret the observed features. However, there have been reports on far-infrared spectra of benzoic acid and its derivatives [10], glucose and uric acid [11], DNA and bovine serum albumin [12], retinal isomers [13] and of nucleobases of DNA $[14,15]$. In each case, the spectral features of crystalline samples in the low-frequency region $\left(<100 \mathrm{~cm}^{-1}\right)$ are associated with collective vibrational modes of molecules mediated by hydrogen bonded networks.

In this article, we present the far-infrared absorption spectra of a range of monosaccharides (with a hexopyranose structure) and disaccharide molecules. Specifically, the temperature dependent spectra of low-frequency vibrational modes in D-glucose, D-mannose, D-galactose and D-fructose (monosaccharides) are discussed together with spectra for maltose and lactose (disaccharides).

\section{Experimental}

All bio-molecules were purchased from Sigma-Aldrich Co. and used without further purification. Samples, in the form of polycrystalline powder, were mixed with polyethy- 


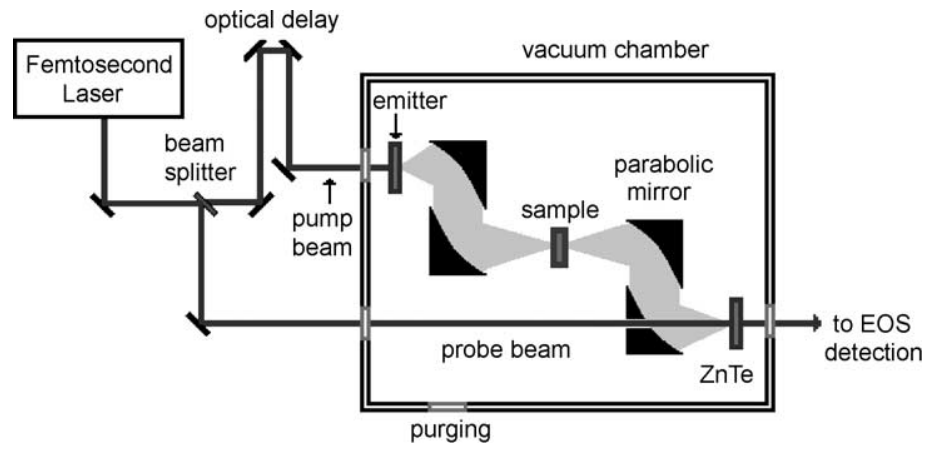

Fig. 1. Terahertz time domain spectroscopy apparatus. The whole generation and detection system was purged with dry nitrogen in order to minimise spectral features owing to water absorption.

lene (PET) powder (Lot 17410AO-061) in a mass ratio of 8:1 (PET: sample) and made into pellets with thicknesses between 1.2 and $1.4 \mathrm{~mm}$. Since PET is nearly transparent [15] in the terahertz range, it is an ideal matrix material. Poly-crystallanity of sugar samples was confirmed by X-ray diffraction measurements. All crystals were crushed well before making the pellets in order to ensure that the particles were of sub-micron size, and that the observed features were not a result of Rayleigh and/or Mie scattering. Indeed, some of the particles have sizes of a few tens of nanometers, as confirmed by environmental SEM measurements.

The experimental apparatus for terahertz transmission measurements is illustrated in Fig. 1 and has been discussed in detail elsewhere $[9,16]$. In brief, optical excitation was achieved by a diode laser pumped Ti:Sapphire laser, emitting $12 \mathrm{fs}$ pulses centred at $790 \mathrm{~nm}$ wavelength, and operating at a $76 \mathrm{MHz}$ repetition rate. A $10 \%$ beam splitter separated the pulses into two beams, a pump beam (90\%) used for generation and a probe beam (10\%) for detection. Generation was by photo-excitation of a $400 \mu \mathrm{m}$ wide aperture antenna, comprising a semi-insulating (SI) GaAs substrate and two vacuum deposited $\mathrm{NiCr} / \mathrm{Au}$ electrodes biased up to $80 \mathrm{~V}$. The emitted terahertz pulses were collimated and focused by a series of off-axis parabolic mirrors onto the sample. The transmitted terahertz pulses from the sample were recollimated using another series of off-axis parabolic mirrors and focused onto a $1 \mathrm{~mm}$ thick ZnTe crystal collinear with the optical probe beam. Detection was achieved using an electro-optic sampling (EOS) $[17,18]$ system and a signal-to-noise ratio of $10^{5}$ was achieved using a lock-in detection scheme. The optical delay, between pump and probe pulse, was scanned over a distance of $10 \mathrm{~mm}$, allowing both phase and amplitude information to be obtained. The spectrometer had a useful bandwidth of $0.1-3.0 \mathrm{THz}$ and a spectral resolution of better than $15 \mathrm{GHz}$. The spectroscopic information was obtained by comparing the terahertz electric field recorded with and without a sample in the beam path. For the low temperature measurements, samples were mounted in a cryostat equipped with mylar windows. The terahertz beam path was always purged with dry nitrogen to minimise absorption by water vapour.

\section{Results and discussion}

Fig. 2a shows terahertz absorption spectra for D-glucose both at room temperature $(295 \mathrm{~K})$ and $4 \mathrm{~K}$. Distinct absorption peaks are seen in the spectral range $0.1-3.0 \mathrm{THz}$ together with small oscillations at low frequencies which are caused by etalon effects resulting from terahertz reflections at the surfaces of the sample. At $4 \mathrm{~K}$, the absorption peaks become narrow and well-resolved with an increase in peak intensity. The peak position also exhibits a (blue) frequency shift. In order to see if the observed resonances are consistent for other hexopyranose structures, we measured far-infrared spectra of D-mannose, D-galactose and Dfructose which are shown in Fig. 2b-d. Each molecule has characteristic resonance peaks and most of these have

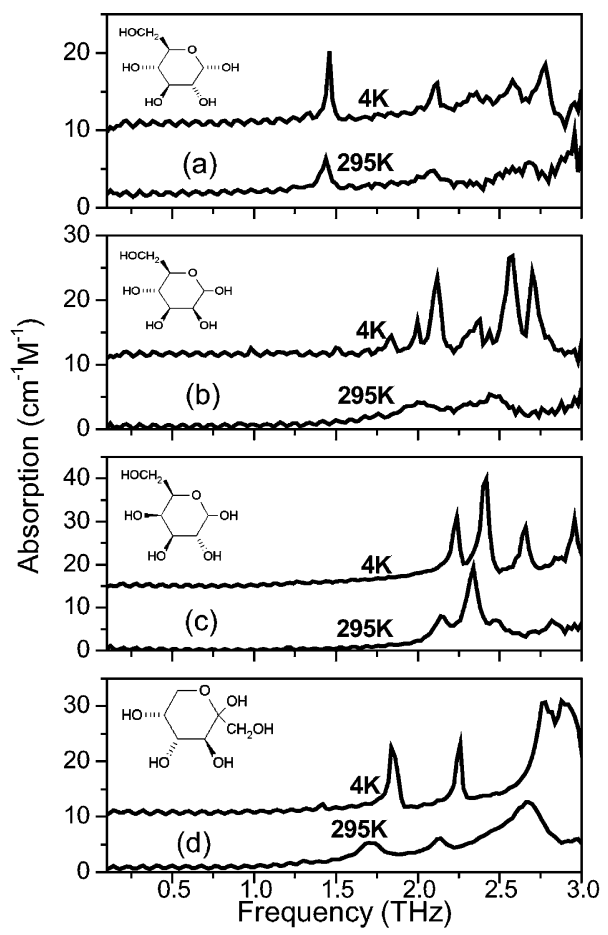

Fig. 2. The far-infrared spectra of (a) D-glucose, (b) D-mannose, (c) Dgalactose and (d) D-fructose measured at 4 and $295 \mathrm{~K}$. The spectra at $4 \mathrm{~K}$ are vertically offset for clarity. 
similar temperature dependent changes to those seen in Dglucose.

The samples used in the experiment, being in polycrystalline powder form, are expected to show intermolecular vibrational modes in this frequency range as well as intramolecular vibrational modes. However, both amorphous and a saturated aqueous solution of D-glucose showed no sharp spectral absorption features in the same spectral range [11]. Thus, it is likely that the observed absorption features are a result of intermolecular vibrational modes mediated by hydrogen bonded networks [14,15], rather than intramolecular modes, the latter being expected to be independent of the degree of erystallinity of the sample. Dauchez et al. [19] and Sekkal et al. [20] have measured the far-infrared absorption in D-glucose and D-galactose in the crystalline state, using FTIR Spectroscopy. On the basis of these results they calculated the vibrational frequencies for D-glucose and Dgalactose, using a modified Urey-Bradley-Shimanouchi force field in combination with a complete intermolecular potential energy function taking into account the van der Waals interactions, the electrostatic interactions, and the contributions of hydrogen bonds. Our observed peaks (at $4 \mathrm{~K}$ ) centered at 1.46 and $2.11 \mathrm{THz}$ for D-glucose, and those (at $4 \mathrm{~K}$ ) centered at 2.23 and $2.65 \mathrm{THz}$ for D-galactose compare well with their experimental measurements and theoretical assignments of the low-frequency translational and rotational lattice vibrational modes in the crystalline structures. These modes, therefore, appear to originate from the collective motion of molecules linked by hydrogen bonds.

Compared to the spectrum of D-glucose, the spectrum of D-mannose has distinct resonances located in the frequency range above $1.7 \mathrm{THz}$. D-Glucose and D-mannose are described as epimers that differ in structural configuration at only one chiral center. Based on comparison of spectra of other epimers and diastereomeric pairs, having one or more than one chiral center, we attribute the unique vibrational signatures of each of these molecules to the change in the potential energy distribution as a result of rearrangement of hydroxyl groups around one or more carbon atoms of the pyranose ring [20].

In our earlier work [21], we investigated the temperature dependent frequency shift by fitting a Bose-Einstein distribution [22] to the absorption features originating from phonons, i.e. intermolecular vibrational modes, mediated by hydrogen bonds:

$v(T)=v_{0}-\frac{A T_{\mathrm{c}}}{\mathrm{e}^{T_{\mathrm{c}} / T}-1}$,

where $v_{0}$ is the center frequency of the vibration mode at $0 \mathrm{~K}$ and $A$ is constant representing the degree of dependence of frequency shift on temperature. $T_{\mathrm{c}}$ is a characteristic temperature related to the energy of the effective phonon.

In Fig. 3, we illustrate the use of this expression by fitting it to the observed peak in D-fructose at $2.26 \mathrm{THz}(4 \mathrm{~K})$. The experimental and best-fit data agree well over the entire

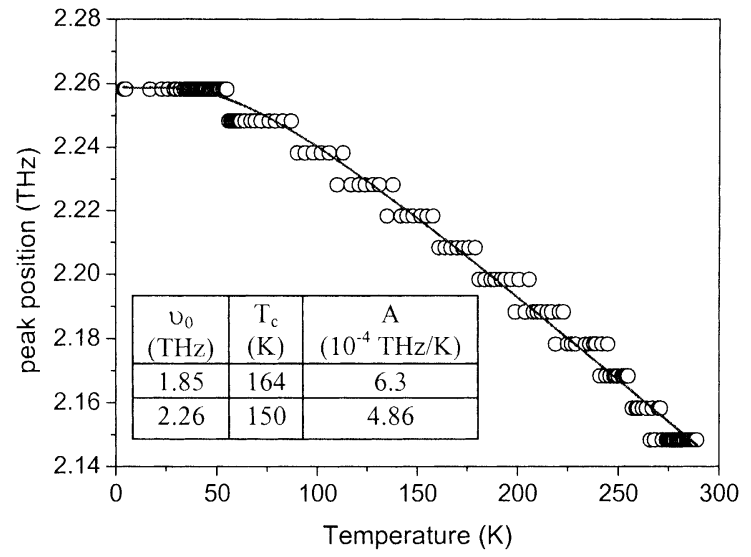

Fig. 3. Temperature dependent shift in the position of the resonance peak centered at $2.26 \mathrm{THz}$ (at $4 \mathrm{~K}$ ) for $\mathrm{D}$-fructose. The open circles are experimental data and the solid line represents the theoretical fit. The inset gives the fitting parameters for the two peaks observed in D-fructose.

temperature range from 4 to $295 \mathrm{~K}$ and the best fitting parameters for the two individual peaks in D-fructose are listed in the inset of Fig. 3. A similar behaviour is also observed for individual features of other molecules, and can be explained by the anharmonicity of the vibrational potential. A temperature dependent change in the distribution of occupied energy states leads to the observed frequency shift and peak broadening/narrowing $[3,4]$.

The dynamics underlying the structural and temperature dependent terahertz absorption were further explored using disaccharide samples which consist of two monosaccharide molecules linked by a glycosidic bond. Absorption spectra of maltose and lactose are depicted in Fig. 4a and b. Although each disaccharide molecule incorporates mono-

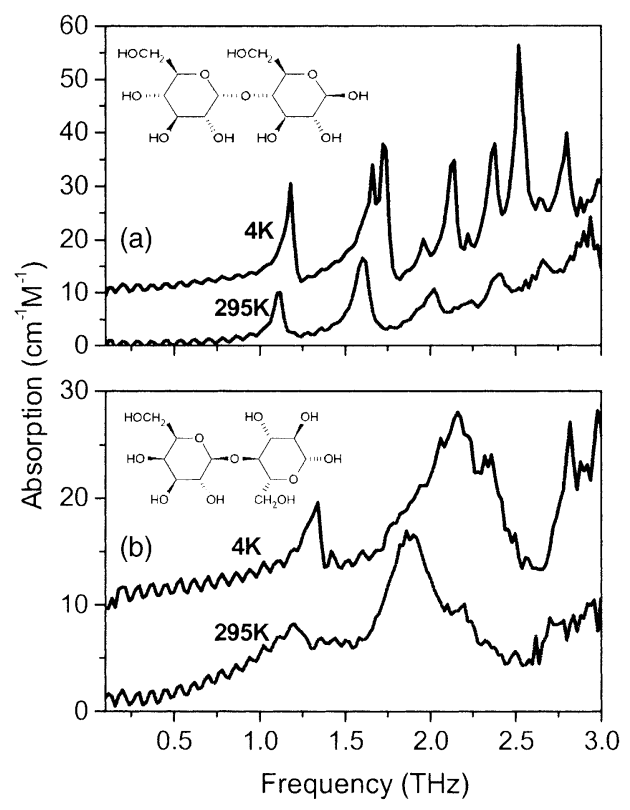

Fig. 4. Far-infrared absorption spectra of (a) maltose and (b) lactose. The spectra at $4 \mathrm{~K}$ are vertically offset for clarity. 


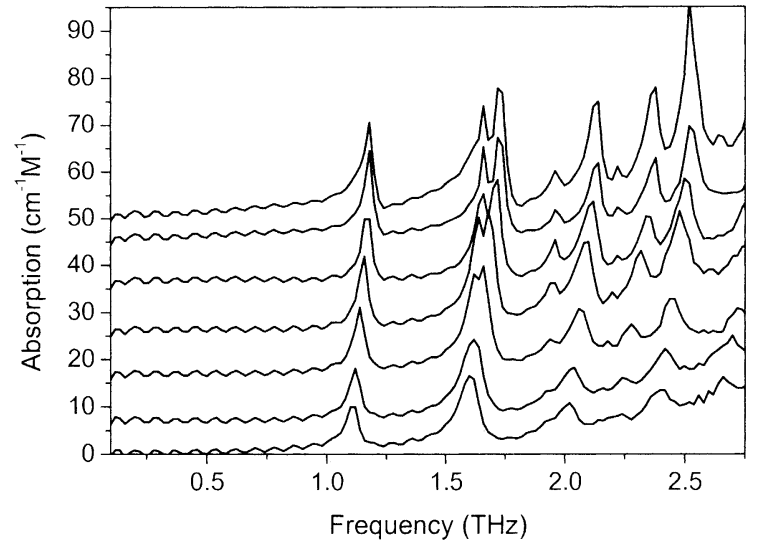

Fig. 5. Temperature dependent frequency shift observed in the absorption spectra of maltose. The spectra were taken at $4 \mathrm{~K}$ (top), 30, 88, 157, 210, 269 and $295 \mathrm{~K}$, respectively, and have been vertically offset for clarity.

saccharide molecular structures, the spectral features are found to be different, with no common spectral features compared to the respective monosaccharide residues. Indeed, many more characteristic resonances are observed at lower frequencies, i.e. $<2.0 \mathrm{THz}$, compared with the monosaccharides. Unlike the monosaccharides, one would expect a significant contribution from the torsional modes of $\mathrm{C}-\mathrm{O}$ bonds, especially around the glycosidic linkage $[23,24]$. We believe that the observed peaks for maltose, which are centered at $1.18,1.65$ and $1.72 \mathrm{THz}$, may be attributed to the lattice vibrational motions together with $\mathrm{C}-\mathrm{O}$ torsional modes [23].

Temperature dependent far-infrared absorption in maltose is illustrated in Fig. 5. Further study is needed to understand fully the observed temperature dependence of the vibrational modes.

Recently, the spectral bandwidth of our terahertz system was extended above $3.0 \mathrm{THz}$ using a modified spectroscopic system [25]. In this system, the generated terahertz waves were collected in a reflection geometry and hence absorption

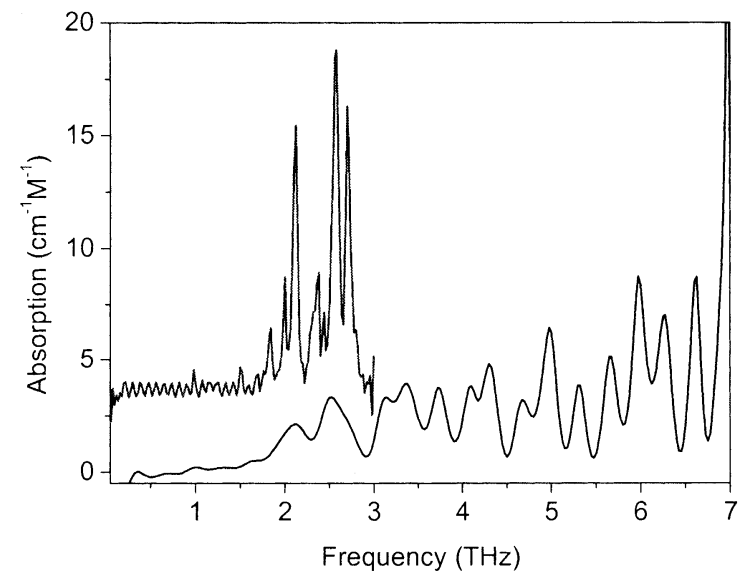

Fig. 6. Broad band absorption spectrum of D-mannose, measured at $4 \mathrm{~K}$ (lower plot). The upper plot depicts the high resolution spectrum $(4 \mathrm{~K})$ recorded with the lower bandwidth spectrometer and is vertically offset for clarity. and dispersion of the terahertz pulse in the GaAs substrate was avoided. By employing this optical geometry, together with a $0.2 \mathrm{~mm}$ thick GaP detector crystal, a spectral bandwidth up to $7.0 \mathrm{THz}$ was achieved. Using this apparatus we have measured the temperature dependent far-infrared absorption of saccharide molecules. However, the strong $\mathrm{THz}$ absorption in the mylar window has limited the usable bandwidth to $6.0 \mathrm{THz}$. In addition, reflections from the window and the detector crystal have limited the spectral resolution, resulting in broad vibrational modes as illustrated in Fig. 6 for D-mannose. Currently the spectral resolution of this system is being optimized to resolve all modes in this frequency range.

\section{Conclusion}

In this work, we have presented far-infrared absorption spectra of a range of monosaccharide molecules, with hexopyranose structures, together with different disaccharides with glycosidic linkage. Using time-domain terahertz spectroscopy in the frequency region between 0.1 and $3.0 \mathrm{THz}$, many distinct vibrational features could be identified as the signatures of inter- and/or intramolecular modes of the hydrogen bonded crystalline structure. These modes are strongly sensitive to the structure and spatial configuration of the molecules. The temperature dependent absorption spectra were recorded in the temperature range of 4-295 K and the observed changes in peak position, intensity and width give information about the dynamics of the temperature dependent potential energy distribution of the hydrogen bonded network.

\section{Acknowledgements}

We acknowledge support from the EPSRC, Royal Society (AGD) and Toshiba Research Europe Ltd (EHL). PCU would like to thank the Association of Commonwealth Universities for financial support.

\section{References}

[1] R. Pethig, D.B. Kell, Phys. Med. Biol. 32 (1987) 933.

[2] A. Markelz, S. Whitmire, J. Hillebrecht, R. Birge, Phys. Med. Biol. 47 (2002) 3797.

[3] T. Uchino, T. Yoko, Science 273 (1996) 480.

[4] A. Xie, Q. He, L. Miller, B. Sclavi, M.R. Chance, Biopolymers 49 (1999) 591.

[5] S.L. Zhang, K.H. Michaelian, G.R. Loppnow, J. Phys. Chem. A 102 (1998) 461.

[6] P.D. Groote, P.G. Rouxhet, J. Devaux, P. Godard, Appl. Spectrosc. 55 (7) (2001) 877.

[7] S. Soderholm, Y.H. Roos, N. Meinander, M. Hotokka, J. Raman Spectrosc. 30 (1999) 1009.

[8] D.K. Buslov, N.A. Nikonenko, N.I. Sushko, R.G. Zhbankov, Spectrochemica Acta Part A. 55 (1999) 229. 
[9] B. Ferguson, X.-C. Zhang, Nat. Mater. 1 (2002) 26 and references therein.

[10] M. Walther, P. Plochocka, B. Fischer, H. Helm, P. Uhd Jepsen, Biopolymers (Biospectroscopy) 67 (2002) 310.

[11] P.C. Upadhya, Y.C. Shen, A.G. Davies, E.H. Linfield, J. Biol. Phys. $29(2 / 3)(2003) 117$.

[12] A.G. Markelz, A. Roitberg, E.J. Heilweil, Chem. Phys. Lett. 320 (2000) 42.

[13] M. Walther, B. Fischer, M. Schall, H. Helm, P. Uhd Jepsen, Chem. Phys. Lett. 332 (2000) 389.

[14] B. Fischer, M. Walther, P. Uhd Jepsen, in: J. M. Chamberlain et al. (Eds.), Proceedings of 2002 IEEE Tenth International Conference on Terahertz Electronics, IEEE, Piscataway, USA, 2002, p. 74.

[15] B. Fischer, M. Waither, P. Uhd Jepsen, Phys. Med. Biol. 47 (2002) 3807.

[16] Y.C. Shen, P.C. Upadhya, E.H. Linfield, A.G. Davies, in: J. M. Chambeilain et al. (Eds.), Proceedings of 2002 IEEE Tenth
International Conference on Terahertz Electronics, IEEE, Piscataway, USA, 2002, p. 165.

[17] Q. Wu, X.-C. Zhang, Appl. Phys. Lett. 67 (24) (1995) 3523.

[18] Q. Wu, X.-C. Zhang, Appl. Phys. Lett. 71 (10) (1997) 1285.

[19] M. Dauchez, P. Derreumaux, G. Vergoten, J. Comp. Chem. 14 (3) (1992) 263.

[20] M. Sekkal, P. Legrand, G. Vergoten, M. Dauchez, Spectrochemica Acta. 48A (7) (1992) 959.

[21] C. Shen, P.C. Upadhya, A.G. Davies, E.H. Linfield, Appl. Phys. Lett. 82 (14) (2003) 2350.

[22] L. Vina, S. Logothetidis, M. Cardona, Phys. Rev. B. 30 (4) (1984) 1979.

[23] M. Dauchez, P. Lagant, P. Derreumaux, G. Vergoten, Spectrochemica Acta. 50A (1) (1994) 105.

[24] D. French, M.K. Dowd, J. Mol. Struct. (Theochem.) 286 (1993) 183.

[25] Y.C. Shen, P.C. Upadhya, E.H. Linfield, H.E. Beere, A.G. Davies, Appl. Phys. Lett. 83 (15) (2003) 3117. 